Farshid Mehrdoust, Hossein Aminikhah, Mohammad Ghamgosar / TJMCS Vol .3 No.4 (2011) 376 - 381

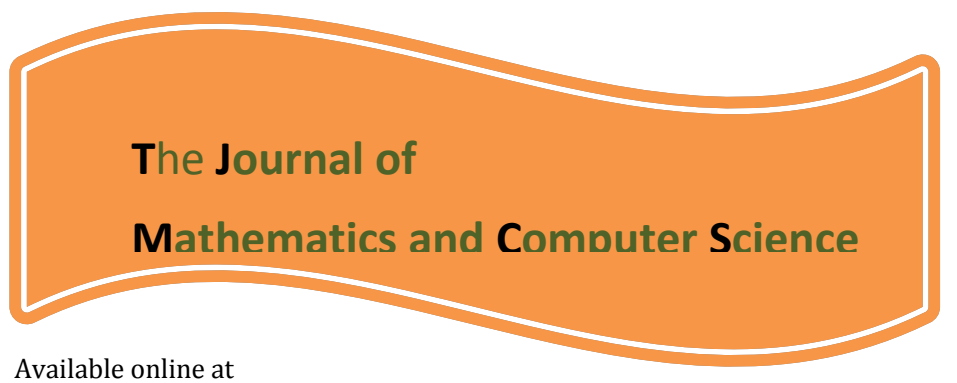

http://www.TJMCS.com

The Journal of Mathematics and Computer Science Vol .3 No.4 (2011) 376 - 381

\title{
Variational Monte Carlo algorithm for solving one dimensional harmonic oscillator problem
}

\section{Farshid Mehrdoust}

Department of Applied Mathematics, Faculty of Mathematical Sciences, University of Guilan, Rasht, Iran, fmehrdoust@guilan.ac.ir

\section{Hossein Aminikhah}

Department of Applied Mathematics, Faculty of Mathematical Sciences, University of Guilan, Rasht, Iran, aminikhah@guilan.ac.ir

\section{Mohammad Ghamgosar}

ghamgosarmohammad@gmail.com

Received: September 2011, Revised: November 2011

Online Publication: December 2011

\begin{abstract}
The goal of this paper is to present an application of variational Monte Carlo method for solving one dimensional harmonic oscillator problem.
\end{abstract}

Keywords: Variational Monte Carlo (VMC); Harmonic oscillator; quantum mechanical systems; Statistical physics.

AMS Subject Classification: 11k45

\section{Introduction}

A basic problem in quantum mechanics is to find the energy eigenstates of quantum mechanical systems. The simplest such system is the quantum harmonic oscillator in one dimension.

The Monte Carlo method is commonly used in physics to simulate complex systems those have 
random nature in statistical physics [1].

\section{Variational Monte Carlo}

The variational method is a powerful tool to estimate the ground state energy of a quantum system, and some of its low lying excited states, when an exact solution cannot be found.When combined with Monte Carlo methods, it can be used on a wide range of atomic and solid state systems, as discussed in the review article by Foulkes et al [2]. The required Monte Carlo techniques for VMC are conceptually simple, but the practical application may turn out to be rather tedious and complex, relying on a good starting point for the variational wave functions. These wave functions should include as much as possible of the inherent physics to the problem, since they form the starting point for a variational calculation of the expectation value of the Hamiltonian H.Given a Hamiltonian $H$ and a trial wave function $\Psi_{T}$ the variational principle states that the expectation value of $\langle H\rangle$ defined through [3]

$$
<H>=\frac{\int \Psi_{T}^{*} H(R) \Psi_{T}(R) d R}{\int \Psi_{T}^{*} \Psi_{T}(R) d R}
$$

is an upper bound to the ground state energy $\mathrm{E}_{0}$ of Hamiltonian $\mathrm{H}$, i.e.

$$
E_{0} \leq<H>
$$

To show this, we note first that the trial wave function can be expanded in the eigenstates of the Hamiltonian since they form a complete set

$$
\Psi_{T}(R)=\sum_{i} a_{i} \Psi_{i}(R)
$$

and assuming the set of eigenfunctions to be normalized, insertion of the latter equation in equation (1) results in

$$
<H>=\frac{\sum_{m n} a_{m}^{*} a_{n} \int \Psi_{T}^{*} H(R) \Psi_{T}(R) d R}{\sum_{m n} a_{m}^{*} a_{n} \int \Psi_{T}^{*} \Psi_{T}(R) d R}
$$

which can be rewritten as

$$
\frac{\sum_{n} a_{n}^{2} E_{n}}{\sum_{n} a_{n}^{2}} \geq E_{0}
$$

In general, the integrals involved in the calculation of various expectation values are multidimensional. Traditional integration methods such as the Gauss Legendre will not be adequate for the computation of the energy of a many-body system. The fact that we need to sample over a multidimensional density and that the probability density is to be normalized by the division of the norm of the wave function, suggests that e.g., the Metropolis algorithm may be appropriate. We could briefly summarize the above variational procedure in the following algorithm. 


\section{Algorithm 1}

1. Construct a trial wave function $\Psi_{T_{i} \alpha}$, for a many-body system consisting of $N$ particles located at positions $R=\left(R_{1}, R_{2}, \ldots, R_{N}\right)$. The trial wave function depends on $\alpha$ variational parameters $\alpha=\left(\alpha_{1}, \alpha_{2}, \ldots, \alpha_{N}\right)$.

\section{Evaluate the expectation value of the Hamiltonian $H$}

$$
<H>=\frac{\int \Psi_{T_{i \alpha} \alpha}^{*}(R) H(R) \Psi_{\mathrm{T}_{j} \alpha}(\mathrm{R}) \mathrm{dR}}{\int \Psi_{T_{i} \alpha}^{*} H(R) \Psi_{\mathrm{T}^{\alpha} \alpha}(\mathrm{R}) \mathrm{dR}}
$$

\section{Vary $\alpha$ according to some minimization algorithm and return to the first step.}

The above loop stops when we reach the minimum of the energy according to some specified criterion. In most cases, a wave function has only small values in large parts of configuration space, and a straightforward procedure which uses homogeneous distributed random points in configuration space will most likely lead to poor results. However, this may suggest that some kind of importance sampling combined with e.g., the Metropolis algorithm may be a more efficient way of obtaining the ground state energy.

The objective is that those regions of configurations space where the wave function assumes appreciable values are sampled more efficiently. The tedious part in a VMC calculation is the search for the variational minimum. A good knowledge of the system is required in order to carry out reasonable VMC calculations. This is not always the case, and often VMC calculations serve rather as the starting point for so-called diffusion Monte Carlo calculations(DMC). DMC is a way of solving exactly the many-body Schrodinger equation by means of a stochastic procedure. A good guess on the binding energy and its wave function is necessary.

Remark 2.1. In the variational Monte Carlo method, a trial wave function $\Psi_{T_{i} \alpha}$ which depends on a set of variational parameters, is carefully chosen. Using our trial wave function we define a new operator, the so-called local energy,

$$
E_{L}(R)=\frac{H \Psi_{T}(R)}{\Psi_{T}(R)}
$$

By defining the following quantal probability distribution

$$
P(R)=\frac{\left|\Psi_{T}(R)\right|^{2}}{\int\left|\Psi_{T}(R)\right|^{2} d R}
$$

we can rewrite the expression for the energy as

$$
<H>=\int P(R) E_{L}(R) d R
$$

and the MC estimator is

$$
<H^{\mathrm{A}}>=\frac{1}{N} \sum_{i=1}^{N} E_{L}\left(x_{i}\right)
$$

The VMC calculation is rather simple, we just generate a large number $\mathrm{N}$ of random numbers corresponding to the Gaussian probability density function and for each random number we compute the local energy according to equation (8). Also, the energy squared through 


$$
<H^{2}>=\int P(R) E_{L}^{2}(R) d R
$$

then MC estimator

$$
<H^{n_{2}}>=\frac{1}{N} \sum_{i=0}^{N} E_{L}^{2}\left(x_{i}\right)
$$

Therefore the variance of the energy as a function of the variational parameter, $\alpha$ equals to

$$
\sigma^{2}=<H^{2}>-<H>^{2}
$$

and we can write

$$
\sigma^{a_{2}}=<H^{\wedge^{2}}>-<H^{a}>^{2}
$$

\section{Numerical results}

Here, we consider the harmonic oscillator in one dimension. The Hamiltonian is

$$
H=-\frac{h^{2}}{2 m} \frac{d^{2}}{d x^{2}}+\frac{k x^{2}}{2}
$$

where $m$ is the mass of the particle and $\mathrm{k}$ is the force constant, e.g., the spring tension for a classical oscillator. In this example we simplify the calculation and choose $m=h=k=1$. The energy of the ground state is then $\mathrm{E}_{0}=1$. The exact wave function for the ground state is

$$
\Psi_{0}(x)=\frac{e^{\frac{-x^{2}}{2}}}{\pi^{\frac{1}{4}}}
$$

but since we wish to illustrate the use of Monte Carlo methods, we choose the trial function

$$
\Psi_{T}(x)=\frac{\sqrt{\alpha}}{\pi^{\frac{1}{4}}} e^{\frac{-x^{2} \alpha^{2}}{2}}
$$

Inserting this function in equation (6) we obtain

$$
E_{L}(x)=\alpha^{2}+x^{2}\left(1-\alpha^{4}\right)
$$

also from (8) we have

$$
<H>=\int_{-\infty}^{+\infty}\left|\Psi_{T}(x)\right|^{2} E_{L}(x) d x
$$

Using simple calculation

$$
<H>=\frac{\alpha^{2}}{2}+\frac{1}{2 \alpha^{2}}
$$

In the following table, results for ground state energy of the harmonic oscillator as a function of the variational parameter $\alpha$. The exact result is for $\alpha=1$ with an energy $\mathrm{E}=1$. The variable $\mathrm{N}$ is the number of Monte Carlo samples. In this calculation we set $N=100000$. 
Farshid Mehrdoust, Hossein Aminikhah, Mohammad Ghamgosar / TJMCS Vol .3 No.4 (2011) 376 - 381

Table 1: Result for ground state energy of the harmonic oscillator as function of the variational Parameter $\alpha$

\begin{tabular}{||l|c|c|c||}
\hline \hline$\alpha$ & Exact solution & MC solution & $\hat{\sigma}$ \\
\hline 0.5 & 2.1250 & 2.1118 & 0.0083 \\
\hline 0.6 & 1.5689 & 1.5689 & 0.0055 \\
\hline 0.7 & 1.2654 & 1.2688 & 0.0035 \\
\hline 0.8 & 1.1012 & 1.1061 & 0.0021 \\
\hline 0.9 & 1.0223 & 1.0233 & 0.0010 \\
\hline 1.0 & 1.0000 & 1.0000 & 0.00 \\
\hline 1.1 & 1.0182 & 1.0174 & 0.0009 \\
\hline 1.2 & 1.0672 & 1.0654 & 0.0017 \\
\hline 1.3 & 1.1409 & 1.1367 & 0.0025 \\
\hline 1.4 & 1.2351 & 1.2255 & 0.0033 \\
\hline 1.5 & 1.3472 & 1.3545 & 0.0040 \\
\hline \hline
\end{tabular}

\section{Concluding Remarks}

We introduced and employed the VMC approach to obtain the numerical ground state energies of the one dimensional harmonic oscillator. The efficiency can be improved by applying quasi random numbers.

\section{References}

[1] Binder K. and D. W. Heermann, 1988. Monte Carlo Simulations in Statistical Physics.

[2] Foulkes W.M.C.,2001. Quantum Monte Carlo simulations of solids, Rev. Mod. Phys.

[3] Pottorf S., Pudzer A., Chou M. Y. and J. E. Hasbun, 1999. The simple harmonic oscillator ground state using a variational Monte Carlo method, Eur. J. Phys. 20(1): 205212. 
Farshid Mehrdoust, Hossein Aminikhah, Mohammad Ghamgosar / TJMCS Vol .3 No.4 (2011) 376 - 381

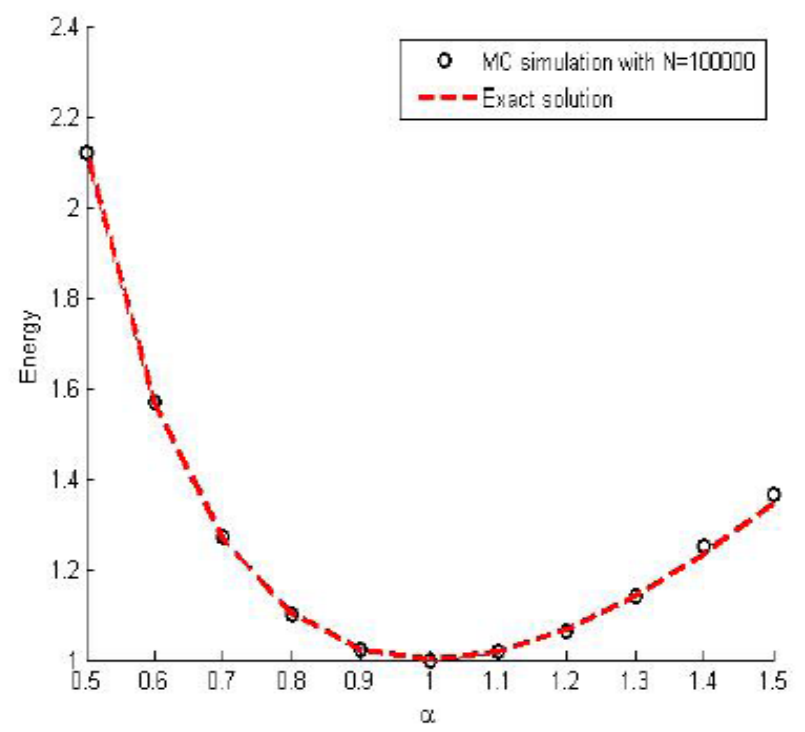

Figure 1: Result for ground state energy of the harmonic oscillator

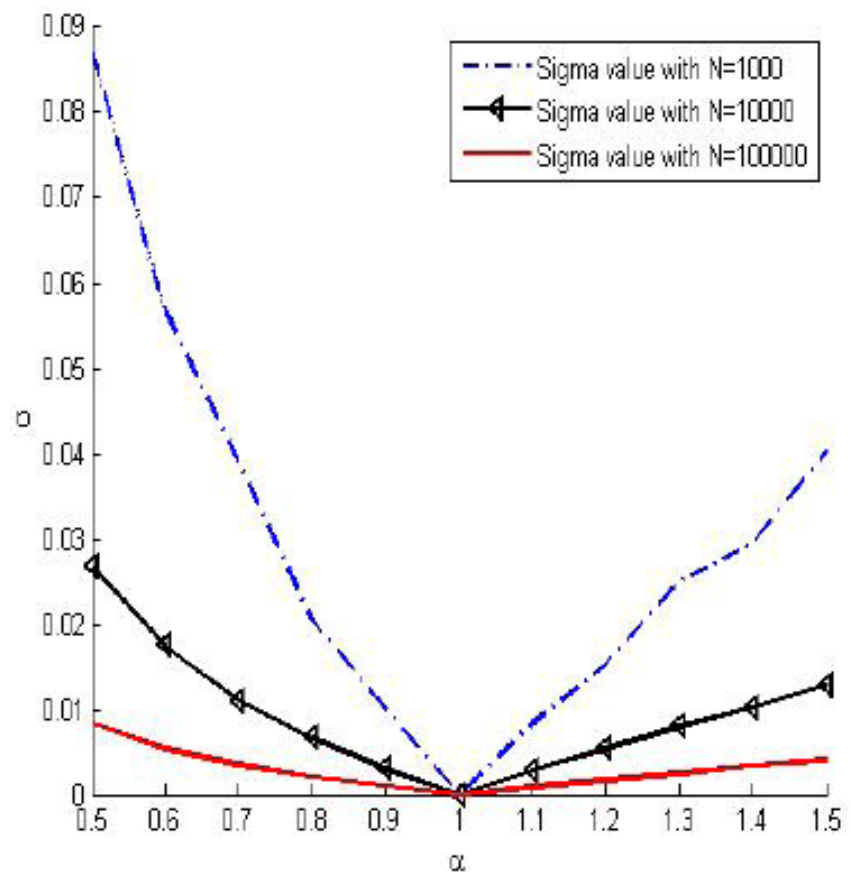

Figure 2: Sigma value for some MC sampling 